\title{
Organic Vapor Jet MicroPrinting of OLED Displays and Lighting Panels
}

\author{
Stephen R. Forrest and Gregory J. McGraw \\ Departments of Physics, Electrical Engineering and Computer Science, \\ and Materials Science and Engineering \\ University of Michigan, Ann Arbor, Michigan 48109
}

\begin{abstract}
Three primary factors govern the cost and performance of organic light emitting diode (OLED) displays that are gaining widespread acceptance for use in mobile electronic appliances. The emitting devices must be of very high efficiency. For this purpose, nearly $100 \%$ internally efficient small molecule electrophosphorescent OLEDs (PHOLEDs) are almost universally used for red, and soon for green emission. The second factor is the transistor "backplane" based primarily on low temperature poly-Si transistor technology, soon to be replaced with metal oxide transistors due to their cost and processing advantages. The last factor forming the "three-legged stool" supporting the widespread use of OLED in large televisions and monitors, is a low cost, fast and high resolution means for pixel patterning. To achieve these metrics, optimal processing methods should combine film growth and patterning into a single step. Practical techniques must be capable of rapid, high resolution patterning, and must also be compatible with high efficiency PHOLED architectures. Finally, the technique must be scalable to Gen 8 substrates and beyond. The most commonly used method is vacuum thermal evaporation using thin metal shadow masks, or alternatively, "scanning shadow masks". However, such masks are susceptible to clogging and are difficult to precisely position over large areas.
\end{abstract}

To meet these challenges, organic vapor jet printing (OVJP) was introduced in our laboratory to enable precise patterning of organic electronic devices, with emphasis on displays and lighting[1]. In OVJP, evaporated organic material is volatilized by, and subsequently entrained in a heated inert carrier gas stream. Vapors from multiple sources, such as the host and dopant in a PHOLED light emitting layer, are then mixed in a common fluidic channel. The heated channel distributes the mixture among an array of nozzles that collimate it into microjets that impinge on a nearby chilled substrate in a vacuum chamber. The organic vapor condenses into well-defined features on the substrate. Desired patterns are generated by moving the substrate relative to the nozzle array. High resolution printing and scalability can be achieved using an array of microfabricated nozzles to simultaneously print multiple PHOLED segments of different colors. In this talk, we show that OVJP is an effective method for printing multicolor PHOLED arrays at the scale required in large area display applications. We find that the separation between adjacent sub-pixels of $50 \mu \mathrm{m}$ (corresponding to $150 \mu \mathrm{m}$ full color pixel separation in a redgreen-blue pixel display) is possible by using a nozzle-tosubstrate separation distance of $10 \mu \mathrm{m}$ along with raised barriers integrated onto the substrate surface. The application of OVJP to display and illumination source printing, along with its potential and limitations will be discussed.

\section{Reference}

[1] M. Shtein, P. Peumans, J. B. Benziger, and S. R. Forrest, "Micropatterning of organic thin films for device applications using organic vapor phase deposition," J. Appl. Phys, vol. 93, p. 4005, 2003. 